EPJ Web of Conferences 60, 12007 (2013)

DOI: $10.1051 /$ epjconf $/ 20136012007$

(C) Owned by the authors, published by EDP Sciences, 2013

\title{
Study of Higgs Production in Fermionic Decay Channels at CMS
}

\author{
Niklas Mohr ${ }^{1, a}$ on behalf of the CMS Collaboration. \\ ${ }^{1}$ Institute for Particle Physics, ETH Zürich, Schafmattstrasse 20, CH-8093 Zürich
}

\begin{abstract}
In these proceedings to the LHCP conference 2013 results are presented on the study of the Higgslike particle at a mass of $125 \mathrm{GeV}$ decaying into final states consisting of either $\tau^{+} \tau^{-}$, or a $b \bar{b}$ quark pair, based on the full statistics of about $24 \mathrm{fb}^{-1}$, collected in 2011 and 2012 at 7 and $8 \mathrm{TeV}$ respectively with the CMS experiment at the Large Hadron Collider. Leptonic and hadronic decay channels for the $\tau$-lepton are included in the search. Different production channels namely gluon fusion, vector boson fusion (VBF) and associated production with $\mathrm{W} / \mathrm{Z}$ bosons have been studied $\tau^{+} \tau^{-}$final states. The $b \bar{b}$ decay channel is studied in VBF as well as in associated production with $\mathrm{W} / \mathrm{Z}$ and top quarks.
\end{abstract}

\section{Introduction}

The recently discovered Higgs-like particle [1, 2] has properties so far consistent with the Standard Model (SM) expectation in terms of spin, parity and couplings [3, 4]. However the excess in fermionic decay channels is still not firmly established. Therefore the study of Higgs properties in fermionic decay channels is of great importance to confirm or disprove the SM nature of the discovered Boson. In the SM with the present dataset of approximately $24 \mathrm{fb}^{-1}$ delivered by the Large Hadron Collider (LHC) and recorded by the CMS experiment [5] the Higgs decay modes into $\tau^{+} \tau^{-}$(denoted by $\tau \tau$ in the following) and $b \bar{b}$ are accessible. For a mass of $125 \mathrm{GeV}$ the branching ratios are about $6.2 \%$ for the $\tau \tau$ decay and about $57 \%$ for the $b \bar{b}$ decay. In the $b \bar{b}$ decay channel the different production modes are very difficult to access and suffer from very different background composition, therefore different analysis techniques are used to extract the Higgs signal in each production mode. The studied production modes cover gluon fusion (only in $\tau \tau$ ), vector boson fusion (VBF) and associated production with $\mathrm{W} / \mathrm{Z}$ bosons and top quarks (only in $b \bar{b}$ ).

In these proceedings the four main analysis channels in fermionic decay modes, $H(\tau \tau), t \bar{t} H(b \bar{b}), \operatorname{VBF}-H(b \bar{b})$ and $V H(b \bar{b})$ are summarized.

\section{$2 H(\tau \tau)$ channel}

In the $\tau \tau$ channel [6] a combined analysis on the $\tau \tau$ invariant mass distribution is employed. The analysis uses the full 7 and $8 \mathrm{TeV}$ dataset of $24.3 \mathrm{fb}^{-1}$. The combined analysis tests the following production modes: gluon fusion, VBF and associated production with a vector boson. Five independent $\tau$-pair final states have been stud-

\footnotetext{
a e-mail: niklas.mohr@cern.ch
}

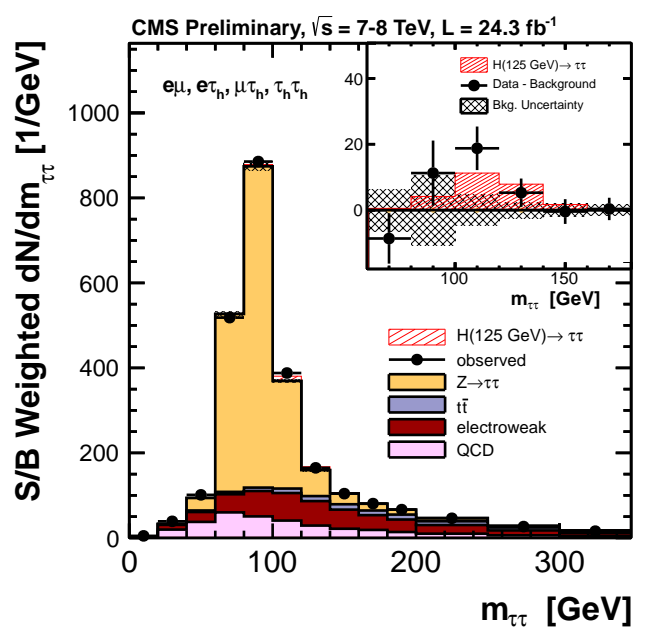

Figure 1. Observed $m_{\tau \tau}$ distributions for a combination of the $\mu \tau_{h}, e \tau_{h}, e \mu$ and $\tau_{h} \tau_{h}$ channels of the $H(\tau \tau)$ analysis. The distributions obtained in each category of each channel are weighted by the ratio between the expected signal and background yields. The insert shows the corresponding difference between the observed data and expected background distributions, together with the expected signal distribution for a standard-model Higgs signal at $m_{\mathrm{H}}=125 \mathrm{GeV}$, with a focus on the signal region.

ied: $\mu \tau_{h}, e \tau_{h}, e \mu$ and $\tau_{h} \tau_{h}$ channels where $\tau_{h}$ denotes a reconstructed hadronic $\tau$ decay. The one-jet category selects primarily signal events with a Higgs boson produced by gluon fusion, or in association with a $W$ or $Z$ boson decaying hadronically. Events in the VBF category are required to have two jets separated by a large rapidity gap, which mainly selects signal events with a Higgs boson produced by VBF and strongly enhances the signal contribution.

The event categories are defined in the following way, using jets within $|\eta|<4.7$. 
- VBF: In the VBF category, two jets with $p_{T}>30 \mathrm{GeV}$ are required to tag the vector-boson fusion Higgsproduction process. The two jets must have an invariant mass $M_{j j}>500 \mathrm{GeV}$ and be separated in pseudorapidity by $\Delta \eta>3.5$. A rapidity gap is defined by requiring no additional jet with $p_{T}>30 \mathrm{GeV}$ between the two tagging jets. In the $e \mu$ channel, the large $t \bar{t}$ background contribution is suppressed by rejecting events containing a $b$-tagged jet of $p_{T}>20 \mathrm{GeV}$.

- 1-jet: Events in this category are required to have at least one jet with $p_{T}>30 \mathrm{GeV}$, not to be part of the VBF event category, and not to contain any $b$-tagged jet with $p_{T}>20 \mathrm{GeV}$. In the $e \tau_{h}$ channel, the large background from $\mathrm{Z} \rightarrow e e+$ jets events in which an electron is misidentified as $\tau_{h}$ is reduced by requiring $M E T>30 \mathrm{GeV}$.

- 0-jet: This category contains all events with no jet with $p_{T}>30 \mathrm{GeV}$, and no $b$-tagged jet with $p_{T}>20 \mathrm{GeV}$. The 0 -jet category is only used to constrain background normalization, identification efficiencies, and energy scales.

Backgrounds are estimated mainly from the data itself. The dominant Drell-Yan production $Z \rightarrow \tau \tau$ is estimated by an "embedding" technique selecting $Z \rightarrow \mu \mu$ events and replacing the muons with simulated $\tau$ decays. Background from $W+$ jets production is estimated in a high-transverse mass control region dominated by the $W+$ jets and extrapolated to the signal region using simulated events. QCD backgrounds are estimated from same-sign events in data. Other small background components are estimated using MC simulations.

The SVFit algorithm is used to improve mass reconstruction in all final states and categories allowing a better separation between signal and background than using only the $\tau \tau$ invariant mass from visible $\tau$ decay products by utilizing the measurement of the missing transverse energy.

Figure 1 shows the combined observed and expected $m_{\tau \tau}$ distributions, weighting all distributions in each category by the ratio between the expected signal and background yields for this category in a $m_{\tau \tau}$ interval containing $68 \%$ of the signal. It also shows the difference between the observed data and expected background distributions, together with the expected distribution for a SM Higgs boson signal with $m_{\mathrm{H}}=125 \mathrm{GeV}$.

The best-fit value for the signal strength combining all channels is $\mu=1.1 \pm 0.4$ at $m_{\mathrm{H}}=125 \mathrm{GeV}$. Fig. 2 shows the expected and observed p-value versus Higgs boson mass. The observed excess is compatible with the presence of a standard model Higgs boson of mass $m_{\mathrm{H}}=125 \mathrm{GeV}$, for which the local significance is $2.85 \sigma$.

\section{$3 t \bar{t} H(b \bar{b})$ channel}

In the $t \bar{t} H(b \bar{b})$ channel [7] the events are classified based on the decay of the associated top quarks: lepton+jets and dilepton events. The dominant background consists of $t \bar{t}$ events. The analysis uses the full $7 \mathrm{TeV}$ and the first $5.1 \mathrm{fb}^{-1}$ of the $8 \mathrm{TeV}$ dataset, so in total an integrated luminosity of $10.1 \mathrm{fb}^{-1}$.

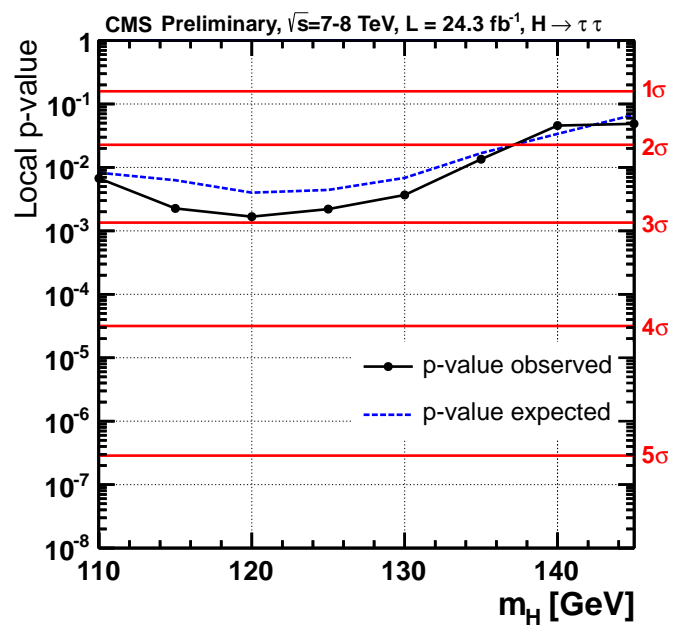

Figure 2. Observed and expected p-value $1-\mathrm{CL}_{b}$, and the corresponding significance in number of standard deviations in the $H(\tau \tau)$ analysis. These results include the search for a SM Higgs boson decaying into a $\tau$ pair and produced in association with a $W$ or $Z$ boson decaying leptonically.

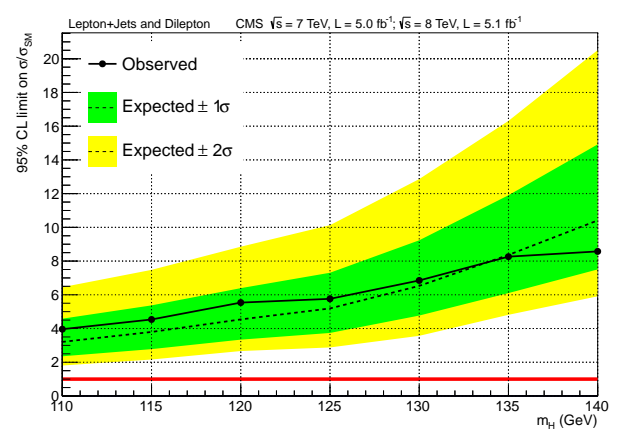

Figure 3. The observed and expected $95 \%$ CL upper limits on the signal strength parameter for lepton+jets and dilepton channels combined in the $t \bar{t} H(b \bar{b})$ channel.

To increase the sensitivity of the analysis selected events are separated into different categories based on the number of jets and $b$-tags. For lepton+jets events, the following seven categories are used: $\geq 6$ jets +2 b-tags, 4 jets +3 b-tags, 5 jets +3 b-tags, $\geq 6$ jets +3 b-tags, 4 jets +4 b-tags, 5 jets $+\geq 4$ b-tags, and $\geq 6$ jets $+\geq 4$ b-tags. For dilepton events, only two categories are used: 2 jets + 2 b-tags and $\geq 3$ jets $+\geq 3$ b-tags.

Artificial neural networks (ANNs) are used in all categories of the analysis to further discriminate signal from background and improve signal sensitivity. Separate ANNs are trained for each jet-tag category, and the choice of input variables is optimized for each as well. The ANN input variables considered are related to object kinematics, event shape, and the discriminant output from the btagging algorithm. A total of 24 input variables has been considered where only a subset has been used in each category. 
Backgrounds are estimated from MC simulation and the dominant systematic uncertainties originate from the $t \bar{t}+b \bar{b}$ theoretical uncertainty as well as from b-tagging.

The observed (expected) 95\% confidence level (CL) limit on the cross section for Higgs boson production in association with top-quark pairs for masses from 110-140 $\mathrm{GeV}$, have been derived using the $7 \mathrm{TeV}$ and $8 \mathrm{TeV}$ samples (Fig. 3). No significant excess is found and the limit at a Higgs boson mass of $125 \mathrm{GeV}$ is 5.8 (5.2) times the standard model expectation.

\section{VBF- $H(b \bar{b})$ channel}

In the VBF- $H(b \bar{b})$ channel [8] events are characterized by two forward quark jets and two $b$-jets originating from the decay of the Higgs boson. The by far dominant background consists of purely QCD multi-jet events which already were reduced at the trigger level. Data at a center of mass energy of $8 \mathrm{TeV}$ corresponding to an integrated luminosity of $19 \mathrm{fb}^{-1}$ have been analyzed. The events are selected by requiring four jets with $p_{T}>$ $85,70,60,40 \mathrm{GeV}$. The four jets are ordered in pairs labeled "bb" and "qq" alternatively with b-tag ordering, where the "qq" pair is made with least b-tagged jets, and with $\eta$ ordering, where the "qq" pair is the most $\eta$ separated jet pair. For both orderings the event selection further requires $m_{\mathrm{qq}}>300 \mathrm{GeV}$ and $\Delta \eta_{\mathrm{qq}}>2.5$. Finally, to remove the large QCD contribution of back-to-back $b b$ pairs, events are required to satisfy $\Delta \phi_{\mathrm{bb}}<2$, for the b-tag ordered jet pair only.

To further identify if the less b-tagged jet pair among the four leading jets is likely to originate from the hadronization of a light (u,d,s-type) quark, as for signal VBF tagging jets, or from gluons, as is more probable for jets produced in QCD processes, a quark-gluon discriminator has been applied to the b-tag sorted "qq" candidate jets.

The signal extraction is performed on the $b b$ pair invariant mass distribution. To improve the mass resolution a neural network regression technique is employed. The sensitivity of the analysis within pre-selection is improved by classifying the events based on a neural network using the characteristics of the "qq" jet pair in order not to bias the $b b$ pair invariant mass distribution.

Data are categorized in four categories and a polynomial of fifth degree is used to model the QCD continuum background. A fit of the $b b$ pair invariant mass distribution in the most sensitive category is shown in Fig. 4.

To validate the search strategy the $m_{b b}$ fit is performed in the same way as for the Higgs boson search, but trying to extract $\mathrm{VBF} Z$ production with $Z \rightarrow b \bar{b}$. The data yields $2844 \pm 1127$ events in the $Z$ peak, i.e. a fit of the $\mathrm{Z}$ peak with a significance of 2.5 standard deviations, in agreement with expectations derived using Monte Carlo pseudo experiments.

Based on all four categories upper limits, at the $95 \%$ confidence level, on the production cross section times the branching ratio, with respect to the expectations for a standard model Higgs boson, are derived for a Higgs boson in

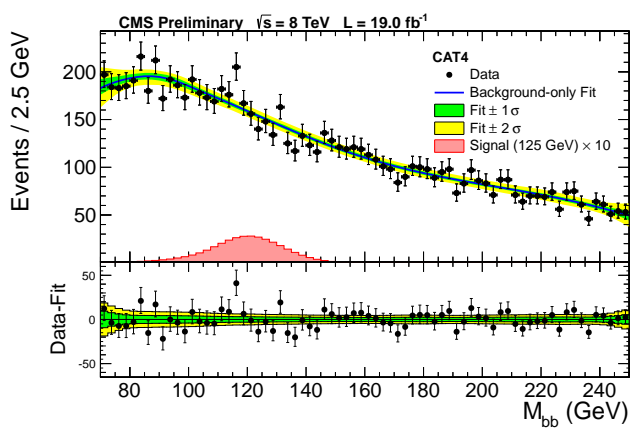

Figure 4. Fit of the background model to the data $m_{b b}$ distribution in the most sensitive category of the VBF- $H(b \bar{b})$ analysis. The top panel show the fitted curve and the amplified distribution of the searched SM signal, in red. The bottom panel shows the data minus fit residuals, together with the fit one and two sigma uncertainty band.

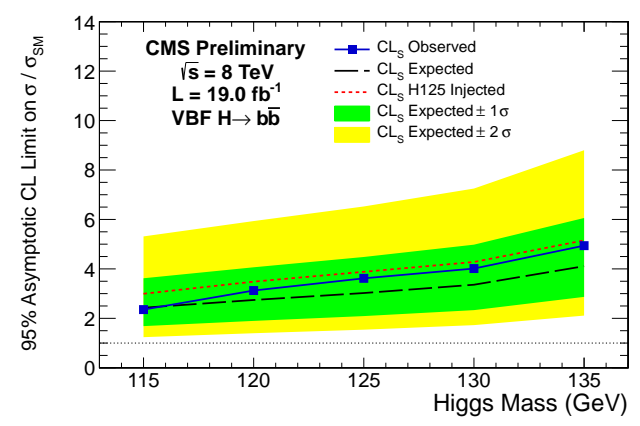

Figure 5. Expected and observed $95 \%$ confidence level limits on the signal cross section in units of the SM expected cross section for the VBF- $H(b \bar{b})$ analysis, as a function of the Higgs boson mass, including all four higher ANN event categories. The limits expected in the presence of a SM Higgs boson with mass $125 \mathrm{GeV}$ are indicated by the dotted curve.

the mass range $115-135 \mathrm{GeV}$. In this range, the expected upper limits in the absence of a signal vary from 2.4 to 4.1 times the standard model prediction, while the corresponding observed upper limits vary from 2.4 to 5.2 (Fig. 5).

At a Higgs boson mass of $125 \mathrm{GeV}$ the expected limit is 3.0 and the observed limit is 3.6. The fitted signal strength is $\mu=\sigma / \sigma_{\mathrm{SM}}=0.7 \pm 1.4$.

\section{$5 V H(b \bar{b})$ channel}

In the $V H(b \bar{b})$ channel [9] the full LHC dataset of $24 \mathrm{fb}^{-1}$ at $7 \mathrm{TeV}$ and $8 \mathrm{TeV}$ has been analyzed. The following modes are included in the search: $\mathrm{W}(\mu \nu) \mathrm{H}, \mathrm{W}(e v) \mathrm{H}$, $\mathrm{W}(\tau v) \mathrm{H}, \mathrm{Z}(\mu \mu) \mathrm{H}, \mathrm{Z}(e e) \mathrm{H}$ and $\mathrm{Z}(v v) \mathrm{H}$, all with the Higgs boson decaying to $b \bar{b}$.

The background processes to $\mathrm{VH}$ production originate from vector-boson+jets $(\mathrm{V}+\mathrm{jets}), t \bar{t}$, single-top and dibosons (VV) production. Except for diboson production, these processes have production cross sections that are several orders of magnitude larger than Higgs boson production. The diboson production cross section is only 


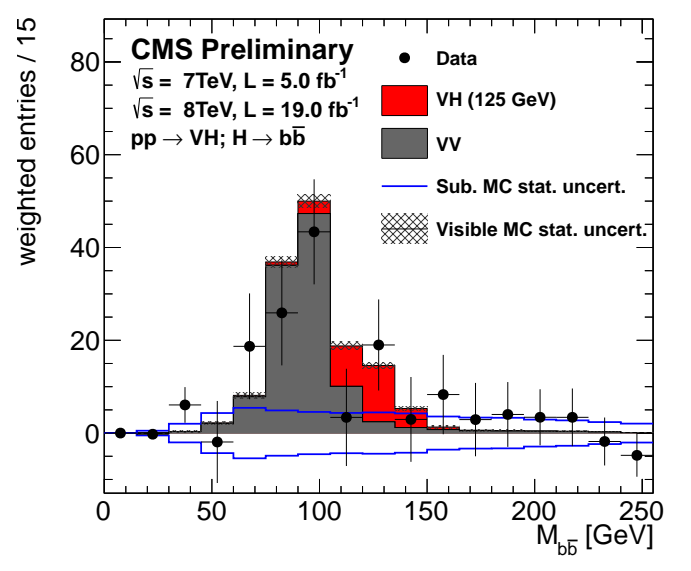

Figure 6. Weighted dijet invariant mass distribution, combined for all channels of the $V H(b \bar{b})$ analysis. For each channel, the relative weight of each $p_{\mathrm{T}}(\mathrm{V})$ bin is obtained from the ratio of the expected number of signal events to the sum of expected signal and background events in a window of $m_{b \bar{b}}$ values between 105 and $150 \mathrm{GeV}$. The expected signal used corresponds to the production of a Higgs boson with a mass of $125 \mathrm{GeV}$. The weight for the highest $p_{\mathrm{T}}(\mathrm{V})$ bin is set to 1.0 and all other weights are adjusted proportionally. All backgrounds, except dibosons, have been subtracted. The solid histograms for the background and the signal are summed cumulatively. The data is represented by points with error bars.

a few times larger than the production cross section for $\mathrm{VH}$ and, given the nearly identical final state for $\mathrm{VZ}$ with $\mathrm{Z} \rightarrow b \bar{b}$, this process provides a benchmark against which the Higgs boson search strategy is tested.

Backgrounds are reduced by requiring a boost of the $p_{T}$ of the vector boson, $p_{\mathrm{T}}(\mathrm{V})$. In that case the Higgs and Vector boson recoil away from each other with a large azimuthal opening angle, $\Delta \phi(\mathrm{V}, \mathrm{H})$, between them. For each mode, different regions of $p_{\mathrm{T}}(\mathrm{V})$ boost are considered. Due to different signal and background composition, each boost region has different sensitivity and the analysis is performed separately in each region. The results from all regions are then combined for each channel. The "low", "intermediate", and "high" boost regions for the $\mathrm{W}(\mu \nu) \mathrm{H}$ and $\mathrm{W}(e v) \mathrm{H}$ channels are $100<p_{\mathrm{T}}(\mathrm{V})<130 \mathrm{GeV}$, $130<p_{\mathrm{T}}(\mathrm{V})<180 \mathrm{GeV}$, and $p_{\mathrm{T}}(\mathrm{V})>180 \mathrm{GeV}$. For the $\mathrm{W}(\tau v) \mathrm{H}$ a single $p_{\mathrm{T}}(\mathrm{V})>120 \mathrm{GeV}$ region is considered. For the $\mathrm{Z}(v v) \mathrm{H}$ channel the "low", "intermediate", and "high" boost regions are $100<p_{\mathrm{T}}(\mathrm{V})<130 \mathrm{GeV}$, $130<p_{\mathrm{T}}(\mathrm{V})<170 \mathrm{GeV}$ and $p_{\mathrm{T}}(\mathrm{V})>170 \mathrm{GeV}$, and for the $\mathrm{Z}(\ell \ell) \mathrm{H}$ channels, the "low" and "high" regions are $50<p_{\mathrm{T}}(\mathrm{V})<100 \mathrm{GeV}$ and $p_{\mathrm{T}}(\mathrm{V})>100 \mathrm{GeV}$.

The Higgs boson mass resolution is improved by applying a bosted decision tree (BDT) regression technique. Using this a further correction, beyond the standard CMS jet energy corrections, for individual $b$-jets improves the $b \bar{b}$ invariant mass resolution by around $15 \%$.

To estimate the backgrounds a set of simultaneous fits is performed to several distributions of discriminating vari-

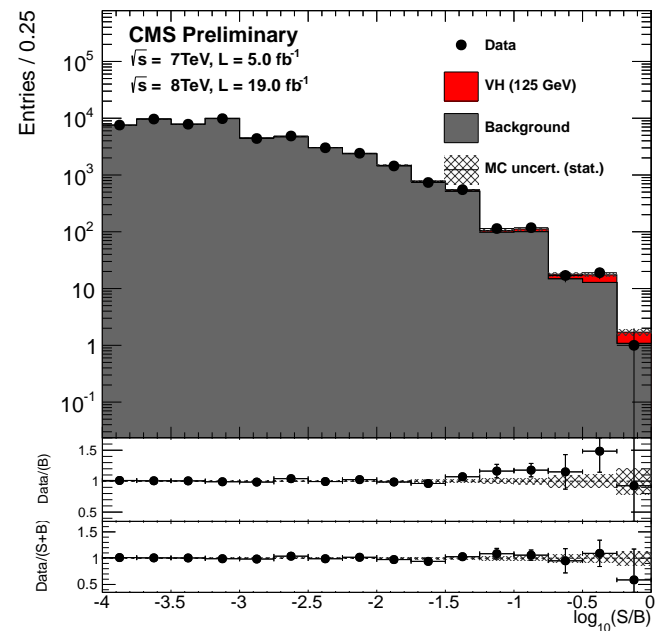

Figure 7. Combination of all BDT discriminants of the $V H(b \bar{b})$ analysis into a single distribution where all events, for all channels, are sorted in bins of similar expected signal-to-background ratio, as given by the value of the output of their corresponding BDT discriminant (trained with a Higgs boson mass of $125 \mathrm{GeV}$ ). The two bottom insets show the ratio of the data to the background-only prediction (above) and to the predicted sum of background plus signal (below).

ables in the control regions, separately in each channel, to obtain consistent scale factors by which the Monte Carlo yields are adjusted.

In each of the $p_{\mathrm{T}}(\mathrm{V})$ categories a boosted decision tree discriminator is used to distinguish signal from background events. Input variables consist of topological information, b-tag related quantities and kinematic quantities. The most important variable in the analysis is the mass of the $b \bar{b}$ system (see Fig. 6), which is also used as input to the BDT. To extract the VH signal a combined fit to all BDT distributions is employed.

As a validation of the multi-variate approach to this analysis, these BDT discriminants are also trained to find diboson signals ( $\mathrm{ZZ}$ and $\mathrm{WZ}$, with $\mathrm{Z} \rightarrow b \bar{b}$ ) rather than the $\mathrm{VH}$ production signal. Using this Diboson BDT the $\mathrm{VZ}$ process with respect to the NLO VZ cross-section, is measured to be $1.19_{-023}^{+0.28}$ with a significance exceeding $7 \sigma$.

Additionally a shape analysis on the mass as a single variable is used to perform a cross-check analysis of the BDT for both VZ and VH signals, yielding consistent results for both cases.

A summary of the BDT analysis is shown in Fig. 7 where all bins in the different categories are shown sorted by their $s / b$. An excess is visible in the most significant bins of the analysis.

Upper limits, at the $95 \%$ confidence level, on the $\mathrm{VH}$ production cross section times the $\mathrm{H} \rightarrow b \bar{b}$ branching ratio, with respect to the expectations for a standard model Higgs boson, are derived for a Higgs boson in the mass range $110-135 \mathrm{GeV}$. In this range, the observed upper limits vary from 1.1 to 3.1 times the SM prediction; the corresponding expected limits vary from 0.7 to 1.5. At a Higgs boson mass of $125 \mathrm{GeV}$ the observed limit is 1.89 while 


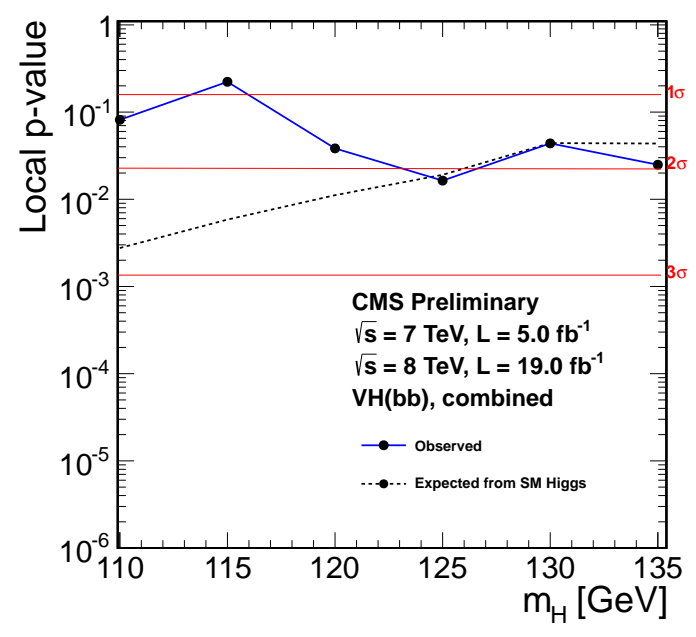

Figure 8. p-values for background fluctuations to account for the observed excess of events in the data in the $V H(b \bar{b})$ analysis.

the expected limit is 0.95 . An excess of events is observed above the expected background with a local significance of 2.1 standard deviations, which is consistent with the expectation from the production of the SM Higgs boson (Fig. 8). The signal strength corresponding to this excess, relative to that of the SM Higgs boson, is $1.0 \pm 0.5$.

\section{Summary}

Four analysis of Higgs boson production in fermionic decay channels have been presented. Different production channels namely gluon fusion, vector boson fusion (VBF) and associated production with $\mathrm{W} / \mathrm{Z}$ bosons have been studied in the $\tau \tau$ mode. The $b \bar{b}$ decay channel has been studied in VBF as well as in associated production with $\mathrm{W} / \mathrm{Z}$ and top quarks. All derived limits, significances of the excesses and signal strength with respect to the SM expectations in the four analysis are summarized in Tab. 1.
Table 1. Observed 95\% CL limits (expected in parentheses), significance and signal strength ( $\mu$-value) with respect to the SM expectation in the four analysis channels.

\begin{tabular}{lccc}
\hline Channel & Limit & Significance & $\mu$-value \\
\hline$H(\tau \tau)$ & $2(0.83)$ & 2.9 & $1.1 \pm 0.4$ \\
$V H(b \bar{b})$ & $1.9(0.95)$ & 2.1 & $1.0 \pm 0.5$ \\
$\operatorname{VBF}-H(b \bar{b})$ & $3.6(3.0)$ & - & $0.7 \pm 1.4$ \\
$t \bar{t} H(b \bar{b})$ & $5.8(5.2)$ & - & - \\
\hline
\end{tabular}

In the $H(\tau \tau)$ and $V H(b \bar{b})$ first hints of a signal are emerging at a significance above $2 \sigma$. Individually non of the channels is able to claim an evidence for Higgs decays into fermions at present.

However, a combination of the $H(\tau \tau)$ and the $V H(b \bar{b})$ analysis yields an observed significance of $3.4 \sigma$ [4] at a Higgs boson mass of $125 \mathrm{GeV}$, which can be interpreted as evidence for decays of the Higgs boson into fermions.

Nevertheless a larger dataset will be needed to firmly establish all Higgs boson decay and production processes in fermionic decay channels. This dataset is expected to be collected at a higher center of mass energy during the LHC Run 2 starting in 2015.

\section{References}

[1] Atlas Collaboration, Physics Letters B 716, 1 - 29 (2012)

[2] CMS Collaboration, Physics Letters B 716, 30 - 61 (2012)

[3] Atlas Collaboration, Atlas conference note ATLASCONF-2013-040, 2013

[4] CMS Collaboration, CMS physics analysis summary HIG-13-005, 2013

[5] CMS Collaboration, Journal of Instrumentation $\mathbf{3}$, S08004 (2008)

[6] CMS Collaboration, CMS physics analysis summary HIG-13-004, 2013

[7] CMS Collaboration, Journal of High Energy Physics 5, 1-47 (2013)

[8] CMS Collaboration, CMS physics analysis summary HIG-13-011, 2013

[9] CMS Collaboration, CMS physics analysis summary HIG-13-012, 2013 